\title{
Materials science aspects of stress corrosion cracking of Russian pipelines
}

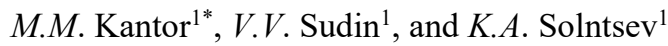 \\ ${ }^{1}$ A.A. Baikov Institute of Metallurgy and Materials Science, Russian Academy of Sciences, Leninskii pr. 49, Moscow, 119334 \\ Russia
}

\begin{abstract}
The work describes the features of stress corrosion cracking (SCC) on Russian gas pipelines. The influence of metal parameters of pipelines on the propagation of SCC is described. The main diagnostic features of SCC used for identification of this type of fracture, the stages of crack growth and the effect of microstructural metal parameters on their propagation are described. Accounting methods for main features crack initiation and growth in order to improve the resistance of the main pipelines metal to SCC

proposed
\end{abstract}

\section{Introduction}

In oil and gas industry, the term stress corrosion cracking (SCC) is used to describe the destruction of pipe metal during long-term operation of main pipelines, which occurs through the slow propagation of cracks. Ordinarily, this kind of destruction occurs on the outer surface of the pipe as a result of simultaneous prolonged exposure of ground electrolytes on the pipeline material, as well as external and internal mechanical stresses appearing during operation of pipelines and pipe production. SCC is one of destruction types, which is characterized by a prolonged growth of subcritical cracks.

As a result of the researches, the existence of two types of SCC, which caused the destruction of pipes of main pipelines in various countries, was established. Depending on the electrochemical properties of the medium in contact with the pipe surface (i.e., depending on whether this medium is acidic or alkaline), they are called SCC in almost neutral $\mathrm{pH}$ and $\mathrm{SCC}$ in high $\mathrm{pH}$ values. The largest number SCC accidents in high $\mathrm{pH}(>$ 9) is recorded on USA pipelines. Carbonates and bicarbonates are substances that stimulate this type of SCC. The destruction of metal pipes due to this SCC type is intergranular.

SCC in almost neutral $(\mathrm{pH}=5 \div 7.5)$ media is realized through transgranular crack propagation [1]. This type of SCC is ordinary for pipelines in Russia and Canada [2]. Neutral environments exhibit low aggressiveness, therefore, analyzing the causes of SCC, all constituent parts of soils must be considered. It is assumed that electrolytes in these soils contain surfaceactive substances, when absorbed on the metal surface of the pipe, reduce its free surface energy and lead to a local decrease in the strength of steel.
Although the electrochemical process is an integral part of SCC, which stimulates the initiation and growth of cracks, under conditions of low soil aggressiveness, SCC cracks of this type significantly propagate through ordinary mechanical destruction.

Resistance to SCC is associated with the structural state of steel, meaning that it is its structure-sensitive property, and depending on the prehistory of steel, its thermomechanical or heat treatment it may be different [3].

\section{Features of SCC diagnostics and identification}

Currently, the main characteristic allowing to identify SCC is the presence of colonies of longitudinal cracks on the outer surface of the pipe. Main role in understanding the process of SCC play non-through cracks, since they characterize the state of the metal in the process of destruction. The depth of different parts of the same nonthrough crack in the pipe metal is different, usually their length is several times greater than the depth [4].

Another important visual characteristic of SCC presence is pipe fracture surface. The stage of stable SCC crack growth necessarily precedes the final destruction of pipe. Therefore, a fracture caused by SCC always consists of at least two zones: stable crack growth and catastrophic destruction.

With the help of microstructural analysis of the metal near the top of the cracks, it is possible to obtain information on the metal state in the process of SCC crack propagation.

Thus, the study of the cracks at the early stage of their formation showed that plastic deformation along cracks insignificant [5], but deep cracks have a blunt tip

\footnotetext{
* Corresponding author: mkantor@imet.ac.ru
} 
with characteristic local plastic deformation of the metal microvolumes in front of it, determined by the local bending of ferritic and pearlitic bands [4].

Since SCC cracks growth rate is small, with the propagation of cracks to a depth of more than 20-30 microns, the crack mouth is filled with corrosion products. Taking into account that the size of ferrite grains in the microstructure of modern pipe steels after controlled rolling is 5-7 $\mu \mathrm{m}$, and in steels after quenching with tempering even 2-3 $\mu \mathrm{m}$ [6], the accuracy of determining the fracture mechanism according to fractographic data seems not quite convincing [7].

The use of modern methods of analysis, such as scanning electron microscopy and electron backscattered diffraction (EBSD), makes it possible to determine reliably the propagation path of a crack as intergranular or transgranular based on information about the crystallographic orientation of grain fragments located on different sides of cracks [5], [8].

\section{Microstructural features of crack propagation}

Earlier was shown that the nucleation of SCC cracks, even under conditions that are characterized by their transgranular growth, occurs at the boundaries of grains and phases [5], [9]. Consequently, at a certain depth under the surface of the metal occurs transition from intergranular crack propagation to transgranular.

On the basis of the SCC cracks of the main pipeline made of steel $17 \mathrm{G} 1 \mathrm{SU}$, by EBSD method, it was shown that microscopic cracks formed on the pipe surface are localized at the grain boundaries and at the phase boundaries. Also, on the surface areas of corrosiondestroyed grain boundaries networks were founded.

Further crack growth is accompanied by a transition to a transgranular propagation path occurring at a depth of about $30 \mu \mathrm{m}$.

The appearance of transgranular growth areas may indicate a reduction in the influence of the corrosion factor in crack growth, which may be a consequence of a decrease in the availability of a corrosive medium in the narrow mouth of the crack near its tip. With increasing crack depth, the increase in mechanical stresses occurs gradually, therefore, the transition from intergranular to transgranular crack growth must be due to crystallographic or geometric parameters of the grains or their boundaries.

The study of the effect of grain boundary parameters on the propagation of SCC cracks showed that low-angle (less than 15 degrees) and special CSL boundaries has the lowest susceptibility for SCC. Also, a strong influence on susceptibility is exerted by the geometrical arrangement of the boundary relative to the direction of the stresses applied to the pipe section with SCC cracks. The boundaries aligned with the direction of crack growth are destroyed much more often than the boundaries extending from the crack profile in the opposite direction to the growth or perpendicular. The study of the character of grain boundaries showed that the boundaries, the misorientation of grains on which is described by a pure slope of lattices, also have increased stability.

It was shown that the transition to the transgranular crack growth path occurs at a depth of about $30 \mu \mathrm{m}$. At the same time, the trigger of such a transition is the emergence of a triple grain junction in the growth path, which consists of boundaries with low susceptibility to $\mathrm{SCC}[10]$

\section{Influence of metal properties on SCC}

Low carbon, low-alloy $17 \mathrm{GS}, 17 \mathrm{G} 1 \mathrm{~S}$ and $17 \mathrm{G} 1 \mathrm{~S}-\mathrm{U}$ steels for several decades have been the basis of Russian main gas pipelines. In the $70 \mathrm{~s}$, the level of production of these steels exceeded 1 million tons per year. Over the years, its basic chemical composition changed. Adjusting the chemical composition in 1968, the introduction of $0.05 \%$ vanadium was allowed.

Besides silicon this steels are deoxidized with aluminum and titanium. Reducing the allowable sulfur content has led to a significant increase in toughness. For pipes with a diameter of 1020-1420 mm, 17G1S steel sheet was supplied in a normalized state. After normalization, steel $17 \mathrm{G} 1 \mathrm{~S}$ has a ferritic-pearlitic microstructure with a significant amount of perlite, which appears in granular form and dispersed among the grains of ferrite.

The basic composition, microstructure and mechanical properties of Russian steel in fact corresponds to imported pipe steel made according to the API-5L standard.

In the last decade, the method of controlled rolling is complemented by the method of accelerated cooling. In this case, the best combination of strength, ductility and toughness is achieved. At the same time, it is necessary to emphasize that, so far, the modes of controlled rolling were chosen only on the basis of optimization of shortterm mechanical properties, by which it is impossible to predict the tendency of pipe steels to SCC.

SCC of metal in Russian pipelines is characterized by the geographical selectivity of its manifestation: in some regions it took place quite often, in others it never happened. This feature of stress corrosion cracking is explained by the need for a sufficiently active corrosive environment.

During the operation of main pipelines in Russia, pipelines from Russian steel of strength categories from $\mathrm{K} 52$ to $\mathrm{K} 60$, and imported ones from X56 to X70, were exposed to SCC. Among the affected to SCC there are both low-alloyed ferritic-pearlitic and low-alloyed highstrength fine-grained steels microalloyed with niobium and vanadium. The latter are sensitive to SCC in the state after controlled rolling as well as after heat treatment (normalization, quench and tempering).

Thus, under the operating conditions of Russian oil and gas pipelines, both conventional ferritic-pearlitic and fine-grained micro-alloyed steels are exposed to SCC. In this case, the latter are sensitive to SCC and in the state after heat treatment, and after controlled rolling.

Laboratory studies of the growth of SCC cracks significantly complicated by their low growth rate. The 
proposed ways to increase the speed of this process usually imply either the use of corrosive media, composition of which differs from natural, or the use of cyclic loads. In the first case, this leads to changes in the mechanism of chemical activation of SCC, for example, in the case of the addition of sulfides, and in the case of cyclic loading, the fracture surface in the region of the SCC crack has grooves usual for fatigue failure.

Testing of the resistance of used in the construction of gas pipelines in the 1970s and 1980s, as well as modern production metal pipes to SCC, showed that more ductile (by relative extension) steel also exhibits a higher resistance to SCC. Resistance to SCC of normalized steels is higher than the steels of controlled rolling production in the $80 \mathrm{~s}$. At the same time, modern steels of controlled rolling with accelerated cooling, being more ductile, exceed normalized steels in resistance to SCC.

Two types of treatment are considered as the most promising types of processing of pipe steels with increased resistance to SCC: thermal improvement, i.e. quenching and tempering and thermomechanical rolling with accelerated cooling.

The process of SCC in the pipes metal under the operating conditions of main gas pipelines significantly differs from laboratory tests, in which the main condition that distinguishes SCC from corrosion fatigue, is the constancy of the applied stresses. During gas pipelines operation, the calculated internal stresses from gas pressure are only the part of actual stresses. Other their constituent parts are technological and operational stresses. At the same time, in individual sections of gas pipelines, the magnitude of operating stresses can be quite significant and variable in time. Suffice it to say that during the inspection of gas pipelines laid in the marshy and watered areas of Western Siberia, some areas of 100-150 meters long were swelling with a maximum deflection of 5 meters or more, while the seasonal variations of the latter were 0.5-0.7 meters [11]. Due to the operational component of the internal stresses, SCC cracks can change direction and not be parallel to the pipe axis, which has been many times observed during the investigation of emergency situations.

The pattern for $\mathrm{pH}$-neutral SCC is the increase in sensitivity to cracking, which is observed with increasing strength of steel. For carbonate cracking in high $\mathrm{pH}$, such a bond is not observed [12]. For SCC in high pH, it is noted that the addition of $\mathrm{Cr}, \mathrm{Ni}$ and $\mathrm{Mo}$ in an amount of from 2 to $6 \%$ increases the resistance to cracking. The authors of most of the works in which they studied SCC of various steel grades agree that the microstructure has a greater influence on the tendency of steel to cracking than the chemical composition.

\section{Conclusion}

A change in the crack propagation path, as well as a change in the degree of plastic deformation of the metal in its tip, indicates a significant change in the mechanism of propagation of SCC cracks during their growth in near-neutral $\mathrm{pH}$ conditions. At the same time, the observed phenomena show that with an increase in the depth of the crack, the contributions of the corrosion factor and the mechanical effect to the crack growth change their ratio. The growth of a crack along the grain boundaries at an early stage of development indicates the predominant participation of corrosion processes in the initiation of a crack. With increasing depth, a decrease in the availability of a corrosive environment and a concentration of stresses at the crack-tip lead to a significant increase in the mechanical factor in the growth of a SCC crack. This leads to a less selective transgranular pathway and a significant increase in the degree of plastic deformation of the metal in the cracktip.

These features determine a significant difference in the growth process of SCC cracks in Russian pipelines from similar processes in the USA and Europe, where most of the cracks are formed under conditions of an alkaline environment and propagation proceeds without changing the mechanism. Nevertheless, it is possible to note some common moments in the nucleation phase, for example, the predominant stability of some grain boundaries, which is determined by their crystallographic features.

Metallurgical parameters such as composition of the metal and non-metallic inclusions play a major role in the crack nucleation stage, and the microstructural parameters such as grain size, the presence of pearlite, toughness and ductility of the metal have a greater influence on the rate of propagation of cracks far from the metal surface.

Thus, taking into account the above features of SCC in a near-neutral environment typical for Russian pipelines to increase the stability of the metal, it is advisable to use steels with increased toughness and ductility, having a uniform microstructure that does not contain areas with increased mechanical stresses, using methods to increase the corrosion resistance of the surface metal layers, including the optimization of crystallographic parameters of the microstructure.

\section{References}

1. B.Y. Fang, A. Atrens, J.Q. Wang, et al, J. Mater. Sci. 38. №. 1. 127-132 (2003)

2. Canadian National Energy Board, MH-2-95 Report of the Inquiry Stress Corrosion Cracking on Canadian Oil and Gas Pipelines, (Calgary, 1996)

3. M.M. Kantor, Proceedings of the meeting of OAO Gazprom «Operating experience and technical diagnostics of gas pipelines with defects of SCC» / Part II. 37-44 (2002)

4. V.G. Antonov, A.B. Arabey, V.N. Voronin et al Stress corrosion cracking of main pipelines. Atlas. (Nauka, Moscow, 2006)

5. M.M. Kantor, V.V. Sudin, V.A. Bozhenov, Scientific and technical collection «Vesti gazovoi nauki» 3 3036 (2016) 
6. L.G. Marchenko, V.I. Stolyarov, A.G. Lyal'kov et al. Steel 11 68-73 (2004)

7. M.A. Stremel' Razrushenie, book 2 (MISiS, Moscow, 2015).

8. D. T. Spencer, M. R. Edwards, M. R. Wenman et al. Corros. Sci. 88 76-88 (2014)

9. R. Chu, W. Chen, S.H. Wang et al. Corrosion. 60 275-283 (2004)

10. M.M. Kantor, V.V. Sudin, K.A. Solntsev, Inorganic Materials 55 409-416 (2019)

11. B.A. Klyuk, V.M. Stoyakov, G.N. Timerbulatov Strength and repair of main pipelines in Western Siberia (Mashinostroenie, Moscow, 1994)

12. R.N. Parkins Overview of intergranular stress corrosion cracking research activities (AGA PRG Report PR-232-9401, 1994) 